\title{
Business Process Models in the Context of Predictive Process Monitoring
}

\author{
Florian Spree ${ }^{1 *}$
}

\begin{abstract}
Predictive process monitoring is a subject of growing interest in academic research. As a result, an increased number of papers on this topic have been published. Due to the high complexity in this research area a wide range of different experimental setups and methods have been applied which makes it very difficult to reliably compare research results. This paper's objective is to investigate how business process models and their characteristics are used during experimental setups and how they can contribute to academic research. First, a literature review is conducted to analyze and discuss the awareness of business process models in experimental setups. Secondly, the paper discusses identified research problems and proposes the concept of a web-based business process model metric suite and the idea of ranked metrics. Through a metric suite researchers and practitioners can automatically evaluate business process model characteristics in their future work. Further, a contextualization of metrics by introducing a ranking of characteristics can potentially indicate how the outcome of experimental setups will be. Hence, the paper's work demonstrates the importance of business process models and their characteristics in the context of predictive process monitoring and proposes the concept of a tool approach and ranking to reliably evaluate business process models characteristics.

Keywords: Business Process Management - Predictive Process Monitoring - Business Process Model Metrics

${ }^{1}$ Computer Science, Technical University of Sofia, Bulgaria

*Corresponding author: Florian.Spree@t-online.de

DOI: http://dx.doi.org/10.22456/2175-2745.106828 • Received: 25/08/2020 • Accepted: 07/12/2020

CC BY-NC-ND 4.0 - This work is licensed under a Creative Commons Attribution-NonCommercial-NoDerivatives 4.0 International License.
\end{abstract}

\section{Introduction}

In recent years, organizations keep on having a high interest to get data-driven insights from day-to-day business operations. One emerging opportunity to improve process performance is to train prediction models. Predictive process monitoring (PPM) exploits process data and aims to predict a userspecified need during runtime. Due to the high complexity of the PPM methodology, empirical evaluation so far has used different setups and benchmarks. In detail, different input data, prediction techniques, domains and prediction goals hinder the comparison. This circumstance makes it difficult to reliably compare the applicability and accuracy of research results. A lack of an exhaustive comparison in the presentation of results is given.

To address the aforementioned gap, this paper focuses on the relevance of business process models. Business process models and their characteristics as one part of the PPM methodology can be assigned to input data. The motivation is to identify if business process models are considered as crucial during academic research and further how business process model characteristics can contribute to academic research. From the authors point of view business process models are vital when documenting, improving, automating, comparing, and predicting existing business processes. As a result, it is necessary to evaluate the business process model characteristics and contextualize it. However, the goal of the work is limited to identify a need of transparency and comparability in the area of PPM rather than identifying influential characteristics. Further, a web-based tool approach gets proposed that can fill the identified gap.

The paper is structured as follows: the second chapter summarizes the main terms related to this work: Business Process Management (BPM), PPM and Business Process Model Metrics. In chapter three, relevant experimental approaches in the area of PPM are described. Chapter four analyses the results based on the research questions derived in chapter three. Chapter five discusses the identified research problem and proposes two approaches to solve the problem: the concept of a web-based business process model metric suite, and to promote a ranking of metrics in the area of PPM. Finally, the paper concludes by summarizing the academic contribution and identifying topics for future work. 


\section{Background}

\subsection{Business Process Management}

BPM is a set of methods, tools and techniques to see how work is performed in an organization [1]. As a central element of contemporary organizations, BPM can support and monitor processes that are for example subject to policies, regulations, and laws. The capability to optimize or support business decisions while running on an enterprise resource planning or workflow system is known as business activity monitoring (BAM) [2]. However, BAM does not provide predictive solutions for an individual running process instance. That is where PPM comes into play. PPM focuses on exploiting generated process data to provide business insights that allow business users to proactively take countermeasures during runtime before they occur.

\subsection{Predictive Process Monitoring}

The PPM methodology aims to predict the future of quantifiable values during a running process execution $[3,4]$. The core of every experimental PPM setup is to build an accurate prediction technique. In the research field of PPM the frameworks proposed by $[5,6]$ are commonly used when performing experiments. In general, the methodology can be divided on a very high level of abstraction into a training and a runtime phase as visualized in figure 1 .

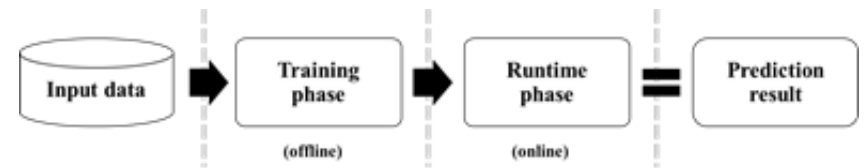

Figure 1. Experimental setup of a predictive process monitoring method

In the first phase, the prediction model is built from finished (offline) input data. Even though the main input data for predictive monitoring methods are finished traces, it further can be classified according to [7] in four different perspectives: the control-flow perspective (concerns the order and relation of activities), the data-flow perspective (concerns the data attributes attached to events), the time perspective (concerns various types of duration such as service times, flow times, waiting times) and the resource/organization perspective (concerns the resource executing the event or corresponding activity). Depending on the type of predictive model it is not necessary to provide all perspectives for training. For example, [5] differentiates between process-aware and non-process aware predictive models. Approaches that are process-aware consider the control-flow perspective as input data explicitly whereas methods that are non-process aware consider the control-flow perspective implicitly. Consequently, after identifying the input data it is necessary to describe an encoding to prepare the relevant information of the process data and finally use the manipulated data to train the predictive model. In general, several predictive models can be considered for training. A common typification is to divide models between classification and regression approaches. The selection of predictive models depends primarily on the type of prediction value (categorical or numeric). Further, the type of predictive model is important for the accuracy assessment: in case of classification methods classification measures such as precision are used. In case of regression methods regression measures such as root-mean squared error are used.

In the second phase, the trained predictive model exploits data corresponding to running (online) traces to predict the outcome during runtime [3]. Based on the prediction result of these traces, the idea is to enable the business to proactively improve process performance and mitigate risks [8]. There are many scenarios where it is useful to have reliable process prediction results, such as predicting compliance violations [9], the remaining sequence of activities [10], or the remaining execution time of a case $[11,12]$. For a better understanding concerning the type of prediction outcome, [13] has identified three main macro-categories: numeric (concerns e.g. the remaining time of an ongoing execution or the costs), categorical (concerns e.g. the class of risk) and the sequence of activities (concerns the prediction of the sequence of future activities).

\subsection{Business Process Model Metrics}

Business process models, regardless whether it is modelled in BPEL [14], EPC [15], BPMN [16] or Petri Nets [12] tend to grow lager and become more complex whenever new business process model characteristics are introduced into an existing process model [17]. In the following, business process model characteristics imply flow objects (events, activities, and gateways) and connecting objects (sequence flow). However, the notation does not indicate if the business process model is provided manually or automatically. Building them manually by users can be very difficult depending on the complexity of the model. Therefore, [18] suggests that if no documented process model exists, or if the process extends across multiple systems, it may be generated automatically through process mining approaches [19, 20] as executed in [21, 22, 23]. Highly complex business process models are error-prone, difficult to understand, to maintain and to manage. Consequently, [24] proposes two approaches to handle complexity and thus keep the highest utility for all stakeholders: First, try to avoid complexity by reducing it. Secondly, control the complexity by metrics.

The paper follows the idea to control the complexity by metrics, meaning measuring business process model characteristics using metrics. Due to the high number of characteristics that contribute to the complexity of a business process model, a variety of valuable metrics have been proposed in the last few decades. This situation is well-known from measuring software complexity. A summary of business process model metrics is provided in table 1 . Table 1 is sorted by year of proposal and provides the following information: The first column references the academic paper for easy access, the second column shows the year of proposal and the last column 
the field of metric. The table structure is also followed in the rest of this subsection.

Table 1. Summary of business process model metrics

\begin{tabular}{lll}
\hline Author & $\begin{array}{l}\text { Year of } \\
\text { Proposal }\end{array}$ & $\begin{array}{l}\text { Business Process Model Met- } \\
\text { ric(s) }\end{array}$ \\
\hline$[25]$ & 2010 & Structural Metrics \\
{$[26]$} & 2010 & Coupling Metrics \\
{$[27]$} & 2009 & Complexity Metrics \\
{$[28]$} & 2007 & Error Metrics \\
{$[29]$} & 2006 & Conceptual Modeling Metrics \\
{$[30]$} & 2006 & Complexity Metrics \\
{$[31]$} & 2006 & Cognitive Complexity Metrics \\
{$[32]$} & 2005 & Complexity Metrics \\
{$[33]$} & 2001 & Complexity Metrics \\
\hline
\end{tabular}

[25] proposed a variety of structural metrics to identify significant predictors for business process model quality. [26] proposes coupling metrics which measure the functional and informational dependencies between the tasks/processes in a business process model. [27] present three complexity metrics that have been implemented in the process analysis tool ProM. [28] provides a set of six metrics to predict errors in business process models. [29] present a suite of metrics for the evaluation of business process models based on the FMESP framework which was developed in order to integrate the modeling and measurement of software processes. [30] defined business process model metrics by gathering insights from software engineering, cognitive science, and graph theory. [31] developed metrics to support the communication between stakeholders by measuring the cognitive complexity of business process models. [32] proposes the metric controlflow complexity (CFC) and discuses to what extent a process is difficult to analyze, understand and explain. [33] summarizes alternative complexity measures discovered in literature.

The result shown in table 1 is similar to research conducted in [34, 35]. Additional, the paper published by [24] presents a classification and an overview of business process metrics and gives an example of implementing these metrics using the ProM tool.

\section{Literature review}

In order to retrieve and select relevant experimental papers in the area of PPM, a literature review was conducted. The purpose is to analyse the PPM research area in a thorough and unbiased manner. The methodology is adapted to a systematic literature review (SLR) as proposed by [36]. The review differs therein that the literature background gets merged from already conducted literature reviews instead of conducting one from the scratch. Thus, the following procedure ensures a rigorous and complete documentation by specifying the research questions, the search protocol and the selection criteria. Below, the research questions (RQ) are formulated, literature background is identified and selection criteria are defined. In line with the selected scope, the paper aims to answer the following research question:

RQ (Methodology): "How do business process models contribute to the research field of predictive process monitoring?"

In line with the main RQ, the paper also answers the following sub-research questions:

Sub-RQ1 (Techniques): "Do predictive techniques affect the relevance of business process models?"

Sub-RQ2 (Metrics): "What business process model characteristics are currently used as a benchmark?"

Next, the review focuses on a broad literature background which should include substantial contributions and the underlying fact that PPM can rely on different setups. In the first step, already conducted literature reviews by researchers in the area of PPM got identified. This approach carries the following benefits: The identified reviews offer different objectives which indicate a broad literature background in the research field and the retrieved studies are already matched against several selection criteria by their authors to confirm their relevance and quality. In addition, the literature reviews were generally applied to well-known electronic literature databases in the field of computer science such as ResearchGate, arXiv, Elsevier, IEEE Xplore and Springer. The result of identified literature reviews is shown in table 2 which is sorted by publication date and provides the following information: The first column references the academic paper for easy access, the second column shows the year of publication, the third column the period of time the SLR has covered and the last column the number of papers identified for each SLR.

Table 2. Conducted SLRs in the area of PPM sorted by publication date

\begin{tabular}{llll}
\hline Author & $\begin{array}{l}\text { Publication } \\
\text { date }\end{array}$ & Years covered & $\begin{array}{l}\text { Number of } \\
\text { Papers }\end{array}$ \\
\hline$[37]$ & 2020 & $2011-2019$ & 39 \\
{$[23]$} & 2019 & $2005-2017$ & 14 \\
{$[38]$} & 2019 & $2005-2017$ & 53 \\
{$[5]$} & 2018 & $2010-2016$ & 41 \\
{$[13]$} & 2018 & $2005-2018$ & 51 \\
\hline
\end{tabular}

In [5] and [38], the most representative time prediction setups of business processes were summarized. Even though both papers had the same intention, the methodology differs. The review by [13] tackles the issue of the high variety of techniques and developed a value-driven framework based on prediction types. [23] presented a categorized collection of outcome-oriented PPM methods to enable researchers to compare methods in a unified setting. Finally, [37] contributed to the knowledge domain by developing a taxonomy of three business use cases to identify relevant papers and thus possibilities for collaboration between academia and industry. In 
conclusion, the identified literature background includes in total 198 papers, 108 excluding duplicates. Duplicates are defined as papers that appear in more than one review result that have the identical title and author(s) [39].

After preparing the literature background, the second step is to select search strings that are relevant to the scope of the paper. These search strings then are applied to the identified literature background to retrieve findings that contain at least one of the strings in the abstract or full text of the paper. The following six search strings are used as keywords. They were derived on the one hand, from the terms introduced in chapter 2 and on the other hand, from terms that can indicate an impact on the research area. Presuming that different authors might use a variety of wordings, several search strings were used to obtain a more exhaustive set of relevant phrases:

$$
\begin{aligned}
& \text { "limitation" - a paper that has mentioned limitations } \\
& \text { "influence" - a paper that has mentioned influencing factors } \\
& \text { "affect" - a paper that has mentioned factors that affect the } \\
& \text { result } \\
& \text { "complexity" - a paper that has mentioned complexity } \\
& \text { "characteristics" - a paper that has mentioned characteristics } \\
& \text { "process model" - a paper that has considered the process } \\
& \text { model }
\end{aligned}
$$

Because the literature background does not encompass areas outside of PPM, the search strings were not combined in a more specific way and applied to all 108 unique papers. One of the threats to validity of the literature review relates to the potential selection bias in the literature background. To minimize it, the result after filtering by search strings is documented on a sufficient level of detail in table 3. To replicate the search, the column "unverified findings" includes all initial findings by search string.

Table 3. Applied search strings to the 108 unique papers

\begin{tabular}{llll}
\hline Search String & $\begin{array}{l}\text { Date of } \\
\text { Search }\end{array}$ & $\begin{array}{l}\text { Unverified } \\
\text { findings }\end{array}$ & $\begin{array}{l}\text { Verified } \\
\text { findings }\end{array}$ \\
\hline limitation & 07.07 .2020 & 30 & 8 \\
influence & 07.07 .2020 & 47 & 15 \\
affect & 07.07 .2020 & 33 & 5 \\
complexity & 07.07 .2020 & 47 & 16 \\
characteristics & 07.07 .2020 & 63 & 28 \\
process model & 07.07 .2020 & 67 & 45 \\
\hline
\end{tabular}

The search was conducted in July 2020 and resulted in 287 unverified findings. An unverified finding stands for one match after conducting the search by search string. Consequently, each search string can occur more than once in a paper. This situation may lead to an increased number of unverified findings with regard to the total amount of papers shown in table 2 . However, to determine if a finding can be considered as valuable, the unverified findings and their context need to be examined. This was accomplished by reading each paragraph containing the search string. To be considered as a verified finding, the content must have a clear link to the business process model and/or its characteristics. The application of the examination resulted in 70 relevant studies and 109 verified results out of 108 unique papers and 287 unverified results.

\section{Assessment of business process mod- els in the context of PPM}

In this section the 109 verified findings that contribute to the formulated RQ get presented. Specifically, the aim is to answer the main RQ (How do business process models contribute to the research field of predictive process monitoring?), Sub-RQ1 (Do predictive techniques affect the relevance of business process models?) and Sub-RQ2 (What business process model characteristics are currently used as a benchmark?). The analysis of the verified findings in the context of the RQ reveals that business process models can be considered in academic research from three different perspectives: First, as input data, secondly as a benchmark and lastly as influencing factor for the prediction outcome.

\subsection{Business process models as input data}

As introduced in section 2.2, input data can be categorized in four different perspectives. One of them is the controlflow perspective which relates to the order of activities and the causal relations between them. Thus, the control-flow perspective is logically linked to business process models. Further, [5] uses the knowledge of business process models in experimental setups to differentiate between prediction models, namely process-aware and non-process aware approaches. Consequently, business process models as input data can affect the selection of prediction models and therefore change the experimental setup assuming that the business process model is the starting point. Pretending the question gets asked the other way around, namely if predictive techniques affect the relevance of business process models Sub-RQ1 can be answered. Considering the categorization by [5] the selection of predictive methods can depend on the availability of business process models as input data in combination with the type of predicted value (categorical or numeric). If the input data is not providing the process model and it can not be generated manually or automatically (for example by using the technique process mining) the experimental setup in terms of prediction models is limited. Whereas as long as the business process model is provided, the experimental setup seems not to be limited at all. A detailed description of process-aware and none process-aware methods and further information are summarized in [5] in table 2 and 3.

\subsection{Business process models as benchmark}

Furthermore, the analysis addresses Sub-RQ2 by answering the question how business models and their characteristics are 
documented and how researcher use the data. The analysis shows that researchers have used business process model data in two ways: First, in a data driven way and secondly in a functional way. The term data driven refers to properties such as available cases and number of executed activities [22, 23]. This means the metrics are business process model related but do not apply to their characteristics. However, researchers have also focused on the functional part of business process models by providing information about the model's characteristics. Characteristics can be on the one hand a simple list of unique objects as documented by $[10,40]$. On the other hand, metrics can be more complex such as the number of sub paths and the largest path length [41].

Although the documentation of business process models and their characteristics in recent research work can be observed, it seems that no standardization for comparability is established. Moreover, even some authors document metrics where it is not clear how to interpret them. In conclusion SubRQ2 can get answered: The general lack of documentation of business process model characteristics and the missing standardization and interpretation lead to the lack of comparison of research results based on business process models. Consequently, no metrics are consciously used as a benchmark yet.

\subsection{Business process models as influencing factor for prediction accuracy}

The most important information of business process models is to specify causal relations between different objects (see section 2.3). For example, the simplest business process models are where activities must be processed in a sequence. Models that contain multiple process variants besides exclusive gateways such as loops, overlapping loops and parallelism increase the number of potentially outcomes of a process and therefore can have a greater impact on the accuracy of prediction. The following observations strengthen the assumption that business process models can influence the prediction accuracy. [41] mentions in his work, when dealing with more complex business process models for example including overlapping loops he has to extend his work. The comment indicates that the complexity of business process models can affect the prediction accuracy. Further, [42] claims that selecting the appropriate path to train a prediction model has an important impact on the model's prediction accuracy. From this assumption can be derived, that the business process model characteristics can have an impact on prediction performance. [43] mention briefly that a high process variability may decrease the precision of predictions. Lastly, [21] describes that loops and parallelism can influence the number of potential future outcomes and therefore need to be considered by the prediction model to reliable predict the likelihood to which each of the future outcome will occur. In summary, it can be observed that researchers are aware of business process model characteristics and their impact on the prediction outcome. However, only general assumptions are mentioned which indi- cates that there is no clear understanding what metric to what extent can influence the prediction accuracy.

In conclusion, the main RQ has been tackled in the context of this work. The result of the analysis according to the number of identified papers strengthens the assumption by a success rate of $76 \%$ (70 relevant papers out 108 papers) that business process models are recognized in the research area of PPM. Further, the analysis reveals that the awareness and potential influence of business process models can be divided in three perspectives: input data, benchmark, and as influencing factor for the prediction outcome. However, the observation further shows that business process models do not contribute to the research field in a reliable and sufficient manner, even they are a firm part of experimental setups. To address this very specific gap, the following chapter describes the area of concern and how it can be improved.

\section{Research problem}

The analysis reveals that the documentation of business process model characteristics differs in the type of applied metrics and is missing a generic nature. This trend is worrying, because metrics can on the one hand provide transparency to compare research results and on the other hand be an indicator for the outcome of research results. For example, business process models that are used as input data for prediction models can further be evaluated in regards of their characteristics. The metrics then can be used as a benchmark for experimental setups and additionally may indicate the accuracy of prediction results.

In order to address the identified research problems, the paper proposes two approaches to solve the problems: First, proposing the concept of a web-based tool to provide a generic view of business process models characteristics and secondly to promote a ranking of metrics to indicate the outcome of prediction accuracy.

\subsection{Business process model metric suite}

Due to the high number of factors that contribute to a business process model's complexity, a single metric cannot consider all needed aspects. Therefore, a common solution is to associate different metrics within a so-called metric suite. A metric suite provides single metrics, which again measure specific aspects of the complexity of a business process model. Providing a tool to support the evaluation of business process models in an easy and complete way should increase the transparency. A very abstract concept of Preva (an acronym for Process evaluation) is shown in figure 2. The idea is to move the state of the art to evaluate business process models characteristics from ad-hoc solutions to a more general metric-suite-based solution approach. The concept supports the evaluation of business process models with various characteristics from different domains such as financial, healthcare, public administration, or insurance.

From an architecture point of view, Preva consists out of four core components that are executed in the following 


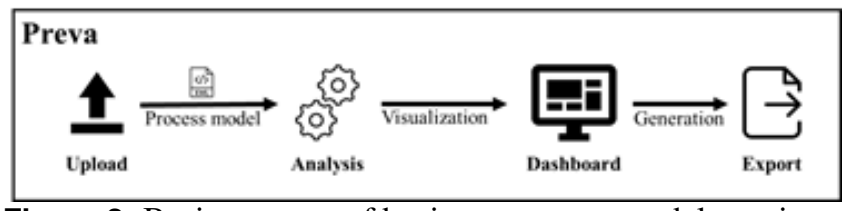

Figure 2. Basic concept of business process model metric suite "Preva"

chronological order: Upload, Analysis, Visualization and Export. The upload component takes the process model as input. Once the business process model is uploaded, the analysis can be triggered and starts to evaluate the uploaded process model. Different metrics shall serve as the basis for analysis. After finishing the analysis, the result is visualized in a dashboard and can be exported as a report.

\subsection{Ranking of metrics}

Business process model metrics can be ranked according to their impact in the context of PPM. The approach enables researchers and practitioners to contextualize metrics and therefore have a indication what the outcome of the prediction models may be. For example, if an experimental setup includes a very complex business process model that includes various overlapping loops and parallelism, chances are high that the prediction accuracy differs from those that only include one exclusive gateway. Providing a ranking of different metrics based on their impact on research results, allows researcher to have an indication how the business process model may influence the prediction accuracy in a positive or negative manner.

\section{Conclusion and future work}

This paper contributes to the knowledge domain by providing a novel and profound analysis to better understand how business process models are currently taken into account in the research area of PPM. As a result, three perspectives were identified. Business process models can be used as input data, as a benchmark and as an influencing factor for the prediction outcome. The analysis further observed that the documentation of business process model characteristics differs in the type of applied metrics and is missing a generic nature. In order to solve the gap, the paper has proposed two approaches: First the concept of a web-based metric suite called preva and secondly to rank metrics based on their context. Though, current research has not yet identified which characteristics of business process models can potentially influence the outcome of experimental results in the area of PPM.

In future work, business process model metrics can be made available in a more sophisticated way by providing a web-based metric suite. It allows the user to access the tool through a web browser and makes metrics available. Consequently, the tool then can be applied automatically in future research activities to provide a benchmark for research results. The tool ensures to evaluate a large amount of business process models in a standardized form with less effort and in a short period of time. This approach is also favoured because of its generic nature which allows it to be used in a high variety of research setups and different contexts. Different context may demand different rankings of metrics. Therefore, ranked metrics can help to gain better insights in the area of PPM. In future work the a prototype of the proposed concept will be developed and influential business process model's characteristics are identified.

\section{References}

[1] DUMAS, M. et al. Fundamentals of Business Process Management. 2. ed. Berlin, Heidelberg: Springer Berlin Heidelberg, 2018. Disponível em:〈http://link.springer.com/10. 1007/978-3-662-56509-4〉.

[2] SCHMIDT, W. Business Activity Monitoring (BAM). In: RAUSCH, P.; SHETA, A. F.; AYESH, A. (Ed.). Business Intelligence and Performance Management. London: Springer London, 2013, (Advanced Information and Knowledge Processing). p. 229-242. Disponível em: 〈http://link. springer.com/10.1007/978-1-4471-4866-1_15〉.

[3] MAGGI, F. M. et al. Predictive Monitoring of Business Processes. In: CAISE: INTERNATIONAL CONFERENCE ON ADVANCED INFORMATION SYSTEMS ENGINEERING, 26., Thessaloniki. Proceedings of the [...]. Cham: Springer, 2014, (Lecture Notes in Computer Science). p. $457-$ 472.

[4] VERENICH, I. A general framework for predictive business process monitoring. In: INTERNATIONAL CONFERENCE ON ADVANCED INFORMATION SYSTEMS ENGINEERING (CAISE), 28., 2016, Ljubljana. Proceedings of the [...]. Aachen: CEUR-WS, 2016. p. 1-9.

[5] MARQUEZ-CHAMORRO et al. Predictive monitoring of business processes: A survey. IEEE Transactions on Services Computing, New York, v. 11, n. 6, p. 962-977, 2018.

[6] METzGER, A. et al. Comparing and Combining Predictive Business Process Monitoring Techniques. IEEE Transactions on Systems, Man, and Cybernetics: Systems, New York, v. 45, n. 2, p. 276-290, fevereiro de 2015. Disponível em: 〈http://ieeexplore.ieee.org/lpdocs/epic03/wrapper.htm? arnumber $=6882809\rangle$.

[7] LEONI, M. de; AALST, W. M. van der; DEES, M. A general process mining framework for correlating, predicting and clustering dynamic behavior based on event logs. Information Systems, Amsterdam, v. 56, p. 235-257, março de 2016. Disponível em: 〈https://linkinghub.elsevier.com/retrieve/pii/ S0306437915001313〉.

[8] SALFNER, F.; LENK, M.; MALEK, M. A survey of online failure prediction methods. ACM Computing Surveys, New York, v. 42, n. 3, p. 1-42, março de 2010. Disponível em: 〈http://portal.acm.org/citation.cfm?doid= 1670679.1670680〉. 
[9] CASTElLANOS, M. et al. A Comprehensive and Automated Approach to Intelligent Business Processes Execution Analysis. Distributed and Parallel Databases, Cham, v. 16 , n. 3, p. 239-273, novembro de 2004. Disponível em: $\langle$ http://link.springer.com/10.1023/B:DAPD.0000031635. $88567.65\rangle$.

[10] EVERMANN, J.; REHSE, J.-R.; FETTKE, P. A Deep Learning Approach for Predicting Process Behaviour at Runtime. In: BPM INTERNATIONAL WORKSHOPS, 2016, Rio de Janeiro. Revised Papers of the [...]. Cham: Springer, 2017. (Lecture Notes in Business Information Processing, v. 281), p. 327-338. Disponível em: 〈http://link.springer.com/10.1007/ 978-3-319-58457-7_24〉.

[11] DONGEN, B. F. van; CROOY, R. A.; AALST, W. M. P. van der. Cycle Time Prediction: When Will This Case Finally Be Finished? In: OTM CONFEDERATED INTERNATIONAL CONFERENCES, COOPIS, DOA, GADA, IS, AND ODBASE, 2008, Monterrey. Proceedings of the [...], Part I. Cham: Springer, 2008, (Lecture Notes in Computer Science, v. 5331). p. 319-336. Disponível em: 〈http: //link.springer.com/10.1007/978-3-540-88871-0_22〉.

[12] ROGGE-SOLTI, A.; WESKE, M. Prediction of Remaining Service Execution Time Using Stochastic Petri Nets with Arbitrary Firing Delays. In: ICSOC: INTERNATIONAL CONFERENCE ON SERVICE-ORIENTED COMPUTING, 11., 2013, Berlin. Proceedings of the [...]. Cham: Springer, 2013, (Lecture Notes in Computer Science, v. 8274). p. 389-403. Disponível em: 〈http://link.springer.com/10.1007/ 978-3-642-45005-1_27〉.

[13] FRANCESCOMARINO, C. D. et al. Predictive Process Monitoring Methods: Which One Suits Me Best? In: BPM: INTERNATIONAL CONFERENCE ON BUSINESS PROCESS MANAGEMENT, 16., 2018, Sydney. Proceedings of the [...]. Cham: Springer, 2018. (Lecture Notes in Computer Science, v. 11080), p. 462-479.

[14] OASIS. Web Services Business Process Execution Language Version 2.0: Oasis standard. Burlington: Organization for the Advancement of Structured Information Standards (OASIS), 2007. Disponível em: 〈http://docs.oasis-open. org/wsbpel/2.0/OS/wsbpel-v2.0-OS.pdf $\rangle$.

[15] WESKE, M. Business Process Management. Berlin, Heidelberg: Springer Berlin Heidelberg, 2012. Disponível em: 〈http://link.springer.com/10.1007/978-3-642-28616-2〉.

[16] OMG. Business Process Model And Notation 2.0 (BPMN). Milford: Object Management Group (OMG), 2011. Disponível em: 〈https://www.omg.org/spec/BPMN/2.0/ About-BPMN/>.

[17] CARDOSO, J. Business Process Control-Flow Complexity. International Journal of Web Services Research, Pensilvânia, v. 5, n. 2, p. 49-76, abril de 2008. Disponível em: 〈http://services.igi-global.com/resolvedoi/resolve.aspx? doi $=10.4018 /$ jwsr.2008040103 $\rangle$.

[18] AALST, W. van der et al. Business process mining: An industrial application. Information Systems, Amsterdam, v. 32, n. 5, p. 713-732, julho de 2007. Disponível em: 〈https: //linkinghub.elsevier.com/retrieve/pii/S0306437906000305〉.

[19] AALST, W. van der et al. Process Mining Manifesto. In: BUSINESS PROCESS MANAGEMENT WORKSHOPS (BPM), 2011, Clermont-Ferrand. Revised Selected Papers, Part I. Cham: Springer, 2012, (Lecture Notes in Business Information Processing, v. 99). p. 169-194. Disponível em: $\langle$ http://link.springer.com/10.1007/978-3-642-28108-2_19〉.

[20] WEIJTERS, A.; AALST, W. van der. Rediscovering workflow models from event-based data using little thumb. Integrated Computer-Aided Engineering, Amsterdã, v. 10, n. 2, p. 151-162, maio de 2003.

[21] LAKSHMANAN, G. T. et al. A markov prediction model for data-driven semi-structured business processes. Knowledge and Information Systems, Cham, v. 42, n. 1, p. 97-126, janeiro de 2015. Disponível em: 〈http://link.springer.com/10. 1007/s10115-013-0697-8>.

[22] TEINEMAA, I. et al. Temporal stability in predictive process monitoring. Data Mining and Knowledge Discovery, Berlin, v. 32, n. 5, p. 1306-1338, 2018.

[23] TEINEMAA, I. et al. Outcome-oriented predictive process monitoring: Review and benchmark. ACM Transactions on Knowledge Discovery from Data, New York, v. 13, n. 2, 2019.

[24] VANDERFEESTEN, R. et al. Quality Metrics for Business Process Models. In: FISCHER, L. 2007 BPM and workflow handbook: Methods, concepts, case studies and standards in business process management and workflow. Lighthouse Point: Future Strategies, 2007. (BPM \& Workflow Handbook Series), p. 179-190.

[25] SÁNCHEZ-GONZÁLEZ, L. et al. Prediction of Business Process Model Quality Based on Structural Metrics. In: INTERNATIONAL CONFERENCE ON CONCEPTUAL MODELING, 29., 2010, Vancouver. Proceedings of the [...]. Cham: Springer, 2010, (Lecture Notes in Computer Science, v. 6412). p. 458-463. Disponível em: 〈http://link.springer. com/10.1007/978-3-642-16373-9_35〉.

[26] KHLIF, W.; ZAABOUB, N.; BEN-ABDALLAH, H. Coupling Metrics for Business Process Modeling. WSEAS Transactions on Computers, World Scientific and Engineering Academy and Society (WSEAS), Stevens Point, v. 9, n. 1, p. 31-41, janeiro de 2010 .

[27] LASSEN, K. B.; AALST, W. M. van der. Complexity metrics for Workflow nets. Information and Software Technology, Amsterdam, v. 51, n. 3, p. 610-626, março de 2009. Disponível em: 〈https://linkinghub.elsevier.com/retrieve/pii/ S0950584908001092 $\rangle$.

[28] MENDLING, J.; NEUMANN, G. Error Metrics for Business Process Models. In: INTERNATIONAL CONFERENCE ON ADVANCED INFORMATION SYSTEMS ENGINEERING, 19., 2007, Trondheim. Proceedings of the [...]. Cham: Springer, 2007. p. 53-56. 
[29] ROLÓN, E. et al. Applying Software Metrics to evaluate Business Process Models. CLEI Electronic Journal, Chile, v. 9, n. 1, junho de 2006. Disponível em: 〈http://192.168.10. 19:8080/cleiej/index.php/cleiej/article/view/310 $\rangle$.

[30] CARDOSO, J. et al. A Discourse on Complexity of Process Models. In: BPM 2006: Business Process Management Workshops. Springer, Berlin, Heidelberg, 2006. p. 117-128. Disponível em: 〈http://link.springer.com/10.1007/ 11837862_13〉.

[31] GRUHN, V.; LAUE, R. Complexity Metrics for Business Process Models. In: INTERNATIONAL CONFERENCE ON BUSINESS INFORMATION SYSTEMS (BIS), 9., 2006, Klagenfurt. Proceedings of the [...]. Klagenfurt, Austria: Gesellschaft für Informatik, 2006. (Lecture Notes in Informatics, v. 85), p. 1-12.

[32] CARDOSO, J. Evaluating the process control-flow complexity measure. In: IEEE INTERNATIONAL CONFERENCE ON WEB SERVICES (ICWS'05), 2005, Orlando. Proceedings of the [...]. Piscataway: IEEE, 2005. Disponível em: $\langle$ http://ieeexplore.ieee.org/document/1530882/〉.

[33] LATVA-KOIVISTO, A. M. Finding a complexity measure for business process models. Espoo: Helsinki University of Technology - Systems Analysis Laboratory, 2001. 26 p. Parecer técnico.

[34] KLUZA, K. Measuring Complexity of Business Process Models Integrated with Rules. In: ICAISC: INTERNATIONAL CONFERENCE ON ARTIFICIAL INTELLIGENCE AND SOFT COMPUTING, 14., 2015, Zakopane. Proceedings, Part II. Cham: Springer, 2015, (Lecture Notes in Computer Science, v. 9120). p. 649-659. Disponível em: 〈http://link.springer.com/10.1007/978-3-319-19369-4_57〉.

[35] MUKETHA, G. et al. A Survey of Business Process Complexity Metrics. Information Technology Journal, Faisalabad, v. 9, n. 7, p. 1336-1344, julho de 2010. Disponível em: 〈http://www.scialert.net/abstract/?doi=itj. 2010.1336.1344).

[36] KITCHENHAM, B. Procedures for Performing Systematic Reviews. Eversleigh: Software Engineering Group, Department of Computer Science, Keele University, 2004. 26 p. Parecer técnico. Disponível em: 〈http://www.inf.ufsc.br/ $\sim$ aldo.vw/kitchenham.pdf $\rangle$.
[37] SOPREE, F. Predictive Process Monitoring: A Use-CaseDriven Literature Review. In: WORKSHOP ON ACADEMY MEETS INDUSTRY IN INFORMATION SYSTEM ENGINEERING (AMISE) CO-LOCATED WITH CAISE'20, 1. 2020, Grenoble. Proceedings of the [...]. Cham: Springer, 2020. v. 40.

[38] VERENICH, I. et al. Survey and Cross-benchmark Comparison of Remaining Time Prediction Methods in Business Process Monitoring. ACM Transactions on Intelligent Systems and Technology, New York, v. 10, n. 4, p. 1-34, julho de 2019. Disponível em: 〈http://dl.acm.org/citation.cfm? doid $=3344873.3331449\rangle$.

[39] KOFOD-PETERSEN, A. How to do a structured literature review in computer science. ResearchGate, Berlin, p. 17, maio de 2015. Version 0.2.

[40] MEHDIYEV, N.; EVERMANN, J.; FETTKE, P. A multistage deep learning approach for business process event prediction. In: IEEE CONFERENCE ON BUSINESS INFORMATICS (CBI), 19., 2017, Thessaloniki. Volume 1: Conference Papers. New York: IEEE Computer Society, 2017. v. 1, p. 119-128.

[41] SPOEL, S. van der; KEULEN, M. van; AMRIT, C. Process Prediction in Noisy Data Sets: A Case Study in a Dutch Hospital. In: SIMPDA: INTERNATIONAL SYMPOSIUM ON DATA-DRIVEN PROCESS DISCOVERY AND ANALYSIS, 2., 2012, Campione d'Italia. Revised Selected Papers. Cham: Springer, 2013. (Lecture Notes in Business Information Processing, v. 162), p. 60-83.

[42] UNUVAR, M.; LAKSHMANAN, G. T.; DOGANATA, Y. N. Leveraging path information to generate predictions for parallel business processes. Knowledge and Information Systems, Cham, v. 47, n. 2, p. 433-461, maio de 2016. Disponível em: 〈http://link.springer.com/10.1007/ s10115-015-0842-7〉.

[43] PIKA, A. et al. Profiling Event Logs to Configure Risk Indicators for Process Delays. In: CAISE: INTERNATIONAL CONFERENCE ON ADVANCED INFORMATION SYSTEMS ENGINEERING, 25., 2013, Valencia. Proceedings of the [...]. Cham: Springer, 2013. (Lecture Notes in Computer Science), p. 465-481. Disponível em: 〈http://link.springer. com/10.1007/978-3-642-38709-8_30 $\rangle$. 